\title{
MAXIMUM PREDICTIVE POWER AND THE SUPERPOSITION PRINCIPLE Q
}

\author{
Johann Summhammer \\ Atominstitut der Österreichischen Universitäten \\ Stadionallee 2, A-1020 Vienna, AUSTRIA
}

\begin{abstract}
In quantum physics the direct observables are probabilities of events. We ask, how observed probabilities must be combined to achieve what we call maximum predictive power. According to this concept the accuracy of a prediction must only depend on the number of runs whose data serve as input for the prediction. We transform each probability to an associated variable whose uncertainty interval depends only on the amount of data and strictly decreases with it. We find that for a probability which is a function of two other probabilities maximum predictive power is achieved when linearly summing their associated variables and transforming back to a probability. This recovers the quantum mechanical superposition principle.
\end{abstract}

\section{Introduction}

Quantum theory is not yet understood as well as e.g. classical mechanics or special relativity. Classical mechanics coincides well with our intuition and so is rarely questioned. Special relativity runs counter to our immediate insight, but can easily be derived by assuming constancy of the speed of light for every observer. And that assumption may be made plausible by epistemological arguments [1]. Quantum theory on the other hand demands two premises. First, it wants us to give up determinism for the sake of a probabilistic view. In fact, this seems unavoidable in a fundamental theory of prediction, because any communicable observation can be decomposed into a finite number of bits. So predictions therefrom always have limited accuracy, and probability enters naturally. More disturbing is the second premise: Quantum theory wants us to give up the sum rule of probabilities by requiring interference instead. However, the sum rule is deeply ingrained in our thought, because of its roots in counting and the definition of sets: Define sets with no common elements, then define the set which joins them all. The number of elements in this latter set is just the sum of the elements of the individual sets. When deriving the notion of probability from the relative frequency of events we are thus immediately led to the sum rule, such that any other rule appears inconceivable. And this may be the reason why we have difficulties accepting the quantum theoretical rule, where probabilities are summed by calculating the square of the sum of the complex square roots of the probabilities. In this situation two views are possible. We may either consider the quantum theoretical rule as a peculiarity of nature. Or, we may conjecture that the quantum theoretical rule has something to do with how we organize

\footnotetext{
${ }^{1}$ This is a slightly revised version of a paper which appeared in Int. J. Theor. Physics 33, 171-178 (1994).
} 
data from observations into quantities that are physically meaningful to us. We want to adopt the latter position. Therefore we seek to establish a grasp of the quantum theoretical rule with the general idea in mind that, given the probabilistic paradigm, there may exist an optimal strategy of prediction, quite independent of traditional physical concepts, but resting on what one can deduce from a given amount of information. We will formulate elements of such a strategy with the aim of achieving maximum predictive power.

\section{Representing Knowledge from Probabilistic Data}

Any investigative endeavour rests upon one natural assumption: More data from observations will lead to better knowledge of the situation at hand. Let us see whether this holds in quantum experiments. The data are relative frequencies of events. From these we deduce probabilities from which in turn we derive the magnitudes of physical quantities. As an example take an experiment with two detectors, where a click is registered in either the one or the other. (We exclude simultaneous clicks for the moment.) Here, only one probability is measurable, e.g. the probablity $p_{1}$ of a click in detector 1 . After $\mathrm{N}$ runs we have $n_{1}$ counts in detector 1 and $n_{2}$ counts in detector 2 , with $n_{1}+n_{2}=N$. The probability $p_{1}$ can thus be estimated as

$$
p_{1}=\frac{n_{1}}{N}
$$

with the uncertainty interval [2]

$$
\Delta p_{1}=\sqrt{\frac{p_{1}\left(1-p_{1}\right)}{N}} .
$$

From $p_{1}$ the physical quantity $\chi\left(p_{1}\right)$ is derived. Its uncertainty interval is

$$
\Delta \chi=\left|\frac{\partial \chi}{\partial p_{1}}\right| \Delta p_{1}=\left|\frac{\partial \chi}{\partial p_{1}}\right| \sqrt{\frac{p_{1}\left(1-p_{1}\right)}{N}} .
$$

The accuracy of $\chi$ is given by the inverse of $\Delta \chi$. With the above assumption we expect it to increase with each additional run, because we get additional data. Therefore, for any $N$, we expect

$$
\Delta \chi(N+1)<\Delta \chi(N) .
$$

However, this inequality cannot be true for an arbitrary function $\chi\left(p_{1}\right)$. In general $\Delta \chi$ will fluctuate and only decrease on the average with increasing $N$. To see this take a theory A which relates physical quantity and probability by $\chi_{A}=p_{1}$. In an experiment of $N=100$ runs and $n_{1}=90$ we get: $\Delta \chi_{A}(100)=.030$. By taking into account the data from one additional run, where detector 2 happened to click, we have $\Delta \chi_{A}(101)=.031$. The differences may appear marginal, but nevertheless the accuracy of our estimate for $\chi_{A}$ has decreased although we incorporated additional data. So our original assumption does not hold. This is worrisome as it implies that a prediction based on a measurement of $\chi_{A}$ may be more accurate if the data of the last run are not included. Let us contrast this to theory $B$, which connects physical quantity and probability by $\chi_{B}=p_{1}{ }^{6}$. With $N$ and $n_{1}$ as before we have $\Delta \chi_{B}(100)=.106$. Incorporation of the data from the additional run leads to $\Delta \chi_{B}(101)=.104$. Now we obviously don't question the value of the last run, as the accuracy of our estimate has increased. 
The lesson to be learnt from the two examples is that the specific functional dependence of a physical quantity on the probability (or several probabilities if it is derived from a variety of experiments) determines whether our knowledge about the physical quantity will increase with additional experimental data, and that this also applies to the accuracy of our predictions. This raises the question what quantities we should be interested in to make sure that we get to know them more accurately by doing more experiments. From a statistical point of view the answer is straightforward: choose variables whose uncertainty interval strictly decreases, and simply define them as physical. And from a physical point of view? Coming from classical physics we may have a problem, as concepts like mass, distance, angular momentum, energy, etc. are suggested as candidates for physical quantities. But when coming from the phenomenology of quantum physics, where all we ever get from nature is random clicks and count rates, a definition of physical quantities according to statistical criteria may seem more reasonable, simply because there is no other guideline as to which random variables should be considered physical.

Pursuing this line of thought we want to express experimental results by random variables whose uncertainty interval strictly decreases with more data. When using them in predictions, which are also expressed by variables with this property, predictions should automatically become more accurate with more data input. Now a few trials will show that there are many functions $\chi\left(p_{1}\right)$ whose uncertainty interval decreases with increasing $N$ (eq.(3)). We want to choose the one with maximum predictive power. The meaning of this term becomes clear when realizing that in general $\Delta \chi$ depends on $N$ and on $n_{1}$ (via $p_{1}$ ). These two numbers have a very different status. The number of runs, $N$, is controlled by the experimenter, while the number of clicks, $n_{1}$, is solely due to nature. Maximum predictive power then means to eliminate nature's influence on $\Delta \chi$. For then we can know $\Delta \chi$ even before having done any experimental runs, simply upon deciding how many we will do. From eq.(3) we thus get

$$
\sqrt{N} \Delta \chi=\left|\frac{\partial \chi}{\partial p_{1}}\right| \sqrt{p_{1}\left(1-p_{1}\right)}=\text { constant }
$$

which results in

$$
\chi=C \arcsin \left(2 p_{1}-1\right)+D
$$

where $\mathrm{C}$ and $\mathrm{D}$ are real constants. The inverse is

$$
p_{1}=\frac{1+\sin \left(\frac{\chi-D}{C}\right)}{2},
$$

showing that the probability is periodic in $\chi$. Aside from the linear transformations provided by $C$ and $D$ any other smooth function $\alpha(\chi)$ in real or complex spaces will also fulfill requirement (5) when equally sized intervals in $\chi$ correspond to equal line lengths along the curve $\alpha(\chi)$. One particular curve is

$$
\alpha(\chi)=\sin \left(\frac{\chi}{2}\right) e^{i \frac{\chi}{2}}
$$

which is a circle in the complex plane with center at $i / 2$. It exhibits the property $|\alpha|^{2}=p_{1}$ known from quantum theory. But note, that for instance the function $\beta=\sin (\chi / 2)$ does not fulfill the requirement that the accuracy only depend on $N$. Therefore the complex phase factor in eq.(8) is necessary [3] [4]. 


\section{Distinguishability}

We have now found a unique transformation from a probability to another class of variables exemplified by $\chi$ in eq.(6). These unique variables always become better known with additional data. But can they be considered physical? We should first clarify what a physical variable is. A physical variable can assume different numerical values, where each value should not only imply a different physical situation, but should most of all lead to a different measurement result in a properly designed experiment. Within the probabilistic paradigm two measurement results are different when their uncertainty intervals don't overlap. This can be used to define a variable which counts the principally distinguishable results of the measurement of a probability. Comparison of that variable to our quantity $\chi$ should tell us how much $\chi$ must change from a given value before this can be noticed in an experiment. Following Wootters and Wheeler [5] 6] the variable $\theta$ counting the statistically distinguishable results at detector 1 in $N$ runs of our above example is given by

$$
\theta\left(n_{1}\right)=\int_{0}^{p_{1}\left(n_{1}\right)} \frac{d p}{\Delta p(p)}=\sqrt{N}\left[\arcsin \left(2 p_{1}-1\right)+\frac{\pi}{2}\right]_{p_{1}=\frac{n_{1}}{N}}
$$

where $\Delta p$ is defined as in eq.(2). When dividing $\theta$ by $\sqrt{N}$ it becomes identical to $\chi$ when in eq.(6) we set $C=1$ and $D=\frac{\pi}{2}$. This illuminates the meaning of $\chi$ : It is a continuous variable associated with a probability, with the particular property that anywhere in its domain an interval of fixed width corresponds to an equal number of measurement results distinguishable in a given number of runs. With Occam's dictum of not introducing more entities than are necessary for the description of the subject matter under investigation, $\chi$ would be the choice for representing physical situations and can rightly be called physical.

\section{A Simple Prediction: The Superposition Principle}

Now we return to our aim of finding a strategy for maximum predictive power. We want to see whether the unique class of variables represented by $\chi$ indicates a way beyond representing data and perhaps affords special predictions. For the sake of concreteness we think of the double slit experiment. A particle can reach the detector by two different routes. We measure the probabilty that it hits the detector via the left route, $p_{L}$, by blocking the right slit. In $L$ runs we get $n_{L}$ counts. In the measurement of the probability with only the right path available, $p_{R}$, we get $n_{R}$ counts in $R$ runs. From these data we want to make a prediction about the probability $p_{t o t}$, when both paths are open. Therefore we make the hypotheses that $p_{t o t}$ is a function of $p_{R}$ and $p_{L}$. What can we say about the function $p_{\text {tot }}\left(p_{L}, p_{R}\right)$ when we demand maximum predictive power from it? This question is answered by reformulating the problem in terms of the associated variables $\chi_{L}$, $\chi_{R}$ and $\chi_{t o t}$, which we derive according to eq.(6) by setting $C=1$ and $D=\frac{\pi}{2}$. The function $\chi_{t o t}\left(\chi_{L}, \chi_{R}\right)$ must be such that a prediction for $\chi_{\text {tot }}$ has an uncertainty interval $\delta \chi_{t o t}$, which only depends on the number of runs, $L$ and $R$, and decreases with both of them. (We use the symbol $\delta \chi_{\text {tot }}$ to indicate that it is not derived from a measurement of $p_{t o t}$, but from other measurements from which we want to predict $p_{t o t}$.) In this way we can predict the accuracy of $\chi_{\text {tot }}$ by only deciding the number of runs, $L$ and $R$. No actual measurements need to have been done. Because 
of

$$
\delta \chi_{t o t}=\sqrt{\left|\frac{\partial \chi_{t o t}}{\partial \chi_{L}}\right|^{2} \frac{1}{L}+\left|\frac{\partial \chi_{t o t}}{\partial \chi_{R}}\right|^{2} \frac{1}{R}}
$$

maximum predictive power is achieved when

$$
\left|\frac{\partial \chi_{t o t}}{\partial \chi_{j}}\right|=\text { constant }, \quad j=L, R .
$$

We want to have a real function $\chi_{t o t}\left(\chi_{L}, \chi_{R}\right)$, and therefore we get

$$
\chi_{t o t}=a \chi_{L}+b \chi_{R}+c
$$

where $a, b$ and $c$ are real constants. Furthermore we must have $c=0$ and the magnitude of both $a$ and $b$ equal to 1 , when we wish to have $\chi_{\text {tot }}$ equivalent to $\chi_{R}$ or to $\chi_{L}$ when either the one or the other path is blocked. So there is an ambiguity of sign with $a$ and $b$. When rewriting this in terms of the probability we get

$$
p_{t o t}=\sin ^{2}\left(\frac{\chi_{L} \pm \chi_{R}}{2}\right)
$$

This does not look like the sum rule of probability theory. Only for $p_{L}+p_{R}=1$ does it coincide with it. We may therefore conclude that the sum rule of probability theory does not afford maximum predictive power. But neither does eq.(13) look like the quantum mechanical superposition principle. However, this should not be surprising because our input were just two real valued numbers, $\chi_{L}$ and $\chi_{R}$, from which we demanded to derive another real valued number. A general phase as is provided in quantum theory could thus not be incorporated. But let us see what we get with complex representatives of the associated variables of probabilities. We take $\alpha(\chi)$ from eq.(8). Again we define in an equivalent manner $\alpha_{L}, \alpha_{R}$ and $\alpha_{t o t}$. From $p_{L}$ we have for instance (from (8) and (7) with $C=1$ and $D=\frac{\pi}{2}$ )

$$
\alpha_{L}=\sqrt{p_{L}}\left(\sqrt{p_{L}}+i \sqrt{1-p_{L}}\right)
$$

and

$$
\Delta \alpha_{L}=\left|\frac{\partial \alpha_{L}}{\partial p_{L}}\right| \Delta p_{L}=\frac{1}{2 \sqrt{L}} .
$$

If we postulate a relationship $\alpha_{t o t}\left(\alpha_{R}, \alpha_{L}\right)$ according to maximum predictive power we expect the predicted uncertainty interval $\delta \alpha_{t o t}$ to be independent of $\alpha_{L}$ and $\alpha_{R}$ and to decrease with increasing number of runs, $L$ and $R$. Analogous to (11) we must have

$$
\left|\frac{\partial \alpha_{t o t}}{\partial \alpha_{j}}\right|=\text { constant }, \quad j=L, R,
$$

yielding

$$
\alpha_{t o t}=s \alpha_{L}+t \alpha_{R}+u
$$

where $s, t$, and $u$ are complex constants. Now $u$ must vanish and $s$ and $t$ must both be unimodular when $p_{t o t}$ is to be equivalent to either $p_{L}$ or $p_{R}$ when the one or the other route is blocked. We then obtain

$$
p_{t o t}=\left|\alpha_{t o t}\right|^{2}=\left|s \alpha_{L}+t \alpha_{R}\right|^{2}=p_{L}+p_{R}+2 \sqrt{p_{L} p_{R}} \cos (\phi)
$$


where $\phi$ is an arbitrary phase factor containing the phases of $s$ and $t$. This is exactly the quantum mechanical superposition principle. What is striking is that with a theory of maximum predictive power we can obtain the general form of this principle, but cannot at all predict $p_{\text {tot }}$ even when we have measured $p_{L}$ and $p_{R}$, because of the unknown phase $\phi$. So we are lead to postulate $\phi$ as a new measurable quantity in this experiment.

\section{Conclusion}

We have tried to obtain insight into the quantum mechanical superposition principle and set out with the idea that it might follow from a most natural assumption of experimental science: more data should provide a more accurate representation of the matter under investigation and afford more accurate predictions. From this we defined the concept of maximum predictive power which demands laws to be such that the uncertainty of a prediction is solely dependent on the number of experiments on which the prediction is based, and not on the specific outcomes of these experiments. Applying this to the observation of two probabilities and to possible predictions about a third probability therefrom, we arrived at the quantum mechanical superposition principle. Our result suggests nature's law to be such that from more observations more accurate predictions must be derivable.

\section{Acknowledgments}

I thank the Austrian Science Foundation (FFW) for financial support of ion double slit experiments (Project P8781-PHY) whose analysis led to this paper.

\section{References}

[1] G. J. Whitrow, The Natural Philosophy of Time, 2nd Ed., (Clarendon Press, Oxford, 1984).

[2] The uncertainty interval is derived from Chebyshev's inequality, e.g. William Feller, An Introduction to Probability Theory and its Applications, 3rd Ed., (Wiley and Sons, New York, 1968), p.233. For reasons of simplicity we are using only the approximate form valid for large $N$.

[3] More details and variables that can be used to represent several probabilities can be found in: J. Summhammer, Found. Phys. Lett. 1, 113 (1988).

[4] The statistical properties of $\chi$ are analyzed in: J. Summhammer, Phys. Lett. A136, 183 (1989).

[5] W. K. Wootters, Phys. Rev. D23, 357 (1981).

[6] J. A. Wheeler, Int. J. Theor. Phys. 21, 557 (1982). 